\title{
PALLIATIVE CARE
}

\section{Analgesia prescribing in the USA —no gain, much pain}

More than two decades ago it was established that patients with cancer frequently receive inadequate analgesic prescribing, despite the publication of pain treatment guidelines. As this is one of the most distressing symptoms experienced by cancer patients and has a tremendous impact on quality of life, Michael Fisch and his colleagues wanted to assess the prevalence of pain and analgesia use in the oncology outpatient setting. They also identified characteristics of patientreported pain, and assessed the adequacy of opioid prescribing, and factors associated with undertreatment of pain.

Data were evaluated from over 3,000 patients from 38 institutions in the USA. Disturbingly, the researchers found that $33 \%$ of cancer patients were undertreated for pain. Of those experiencing moderate or severe pain at initial assessment, $41 \% \mathrm{did}$ not receive an opioid, and $20 \%$ with severe pain did not receive any analgesic. Of the patients who were undertreated at initial assessment, only $31 \%$ received acceptable pain relief during the follow-up visit.
Follow-up analysis revealed that nonHispanic white patients, patients with a poor performance status, and those with advanced cancers receiving treatment were the least likely to have inadequate opioid prescribing. Surprisingly, minority patients were twice as likely as non-Hispanic white patients to receive inadequate treatment for pain on initial assessment and follow up.

As Fisch explains, "these findings related to racial disparity in this study of over 3,000 patients represent a critical observation. These data awaken us to a major opportunity in health care to work harder and smarter to improve cancer pain care for all patients, and to resolve this striking disparity." The reasons for this disparity are not known but might include differences in the attitudes of minority patients about pain-particularly narcotic pain relievers, variation in the patterns of communication between health-care providers and patients, and differences in access to expert pain management and/or patient education information.

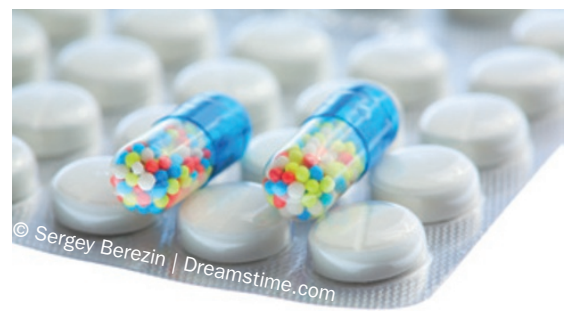

The good news from this study is that there was no disparity related to age or gender. However, cancer patients without advanced disease (survivors) who did not receive treatment for their cancer, were also undertreated for pain.

Fisch notes that immediate ways to improve cancer pain management should include "successful communication with non-oncology specialists and primary care physicians caring for individual patients. This exchange should focus around the patients problem list, goals of treatment, and detailed plan of care."

Lisa Hutchinson

Original article Fisch, M. J. et al. Prospective, observational study of pain and analgesic prescribing in medical oncology outpatients with breast, colorectal, lung, or prostate cancer. J. Clin. Oncol. doi:10.1200/JC0.2011.39.2381 